\title{
Shigella bacteraemia in adults
}

\author{
A.J. Trevett, B.O. Ogunbanjo, S. Naraqi and J.D. Igo
}

Divisions of Medicine and Pathology, Department of Clinical Sciences, University of Papua New Guinea, PO Box 5623, Boroko, Papua New Guinea

Summary: Shigella infections are usually restricted to the intestine. There are few reports of Shigella isolated from the blood and most of these are from children, usually in neonates and the malnourished. In the small number of adult cases of Shigella bacteraemia which have been reported, there appears to be an association with underlying disease and immunosuppression including acquired immunodeficiency syndrome.

We report three adult cases in which Shigella were isolated from blood. Two of these patients made an uneventful recovery whilst the third died. An underlying cause of immunosuppression was suspected in this patient but unproven.

\section{Introduction}

Bacteraemia is rarely documented in shigellosis. This observation might be explained by the fact that blood cultures are not taken routinely from many patients with dysentery or diarrhoea, but studies that have specifically investigated this hypothesis have shown that the rarity of shigellaemia is real. ${ }^{1}$ Bacteraemia appears to occur with greater frequency in neonates, malnourished children and the immunosupressed. Both shigellosis and shigellaemia are less common in adults. This report is based on a retrospective analysis of laboratory and clinical records from a large general hospital over a period of two years. It includes a discussion of the relevant literature to review the significance of finding Shigella spp. in the blood.

\section{Materials and methods}

Port Moresby General Hospital is the main teaching hospital in Papua New Guinea with 600 beds. It is a tertiary referral centre, receiving patients from the whole of National Capital District and Central Province, a catchment area with a population of around 340,000 people (1990 Census).

Blood was collected from all patients with a clinical picture suggestive of septicaemia and immediately cultured in trypticase soya broth for aerobes and into thioglycollate broth for anaerobes. Both were incubated at $36^{\circ} \mathrm{C}$. Subsequent subcultures and follow-up were carried out accord-

Correspondence: A.J. Trevett, M.A., M.B. Ch.B., M.R.C.P., D.T.M. \& H., PO Box 2193, Boroko, NCD, Papua New Guinea.

Accepted: 16 November 1992 ing to standard techniques. ${ }^{2}$ Fresh stool samples were collected from cases of dysentery and diarrhoea, and subjected to standard macroscopic and microscopic examination for parasitic ova, larvae and cysts. Immediate culture was done on conventional media and followed-up according to conven $=$ tional methods. ${ }^{2,3}$ Subsequent detailed biochemicak tests were done including the use of A.P.I. 20E Confirmation of Shigella spp. isolates was made by serogrouping with group- and type-specific antisera from Wellcome laboratories. Antimicrobial susceptibility was carried out by the modified Kirby-Bave disc diffusion method. ${ }^{4}$ The control organism was Escherichia coli ATCC 25922, sensitive to all the tested drugs. Culture of stool and blood samples were done in separate areas of the laboratory by different technicians.

\section{Results}

In the period between July 1989 and June 1991 there was a total of 2,781 stool cultures performed at Port Moresby General Hospital. Of these 42 were positive for Shigella spp., 36 were Shigella flexneri, three Shigella boydii and three Shigella sonnei. There were no isolates of Shigella dysenteriae. During the same period of time 8,730 sets of blood cultures were performed in the hospital. Of these 1,465 were positive $(16.7 \%)$, of which $43 \%$ were enteric organisms. Seven cultures grew Shigella spp., three of which were from adults. Blood cultures were only taken in ten of the patients shown by stool culture to have shigellosis.

The majority of organisms isolated showed multiple antibiotic resistance by disc diffusion testing. Twenty-six out of 37 strains tested $(70 \%)$ 
were resistant to chloramphenicol. Twenty-seven out of 39 isolates tested against ampicillin were resistant $(69 \%)$. Two out of 33 isolates were resistant to gentamicin although the strain isolated from patient 3 was sensitive. Only three out of 39 isolates were resistant to cotrimoxazole $(7.7 \%)$.

The clinical details of the three adult patients in whom Shigella was isolated from blood are described below.

\section{Patient 1}

A 20 year old Melanesian man was admitted to the hospital with a 5 day history of bloody diarrhoea and lower abdominal pain. At admission he was moderately dehydrated. His pulse was $88 /$ minute and regular. His blood pressure was $120 / 80 \mathrm{mmHg}$. He was apyrexial. There was splenomegaly and diffuse abdominal tenderness without guarding. There were no other abnormal findings. Stool cultures grew Shigella flexneri type 1. Blood cultures were taken at admission; two sets of three grew Shigella flexneri. He was treated with intravenous crystalloids and chloramphenicol, $1 \mathrm{~g} 6$ hourly. His symptoms settled and he was discharged on day 5. He was well at follow-up.

\section{Patient 2}

A 25 year old Melanesian man was admitted to the hospital with a 10 day history of bloody diarrhoea and abdominal pain. At admission his pulse was $90 /$ minute, blood pressure was $100 / 60 \mathrm{mmHg}$ with no postural drop. His temperature was $37.2^{\circ} \mathrm{C}$. He had diffuse abdominal tenderness. A stool culture did not grow any pathogen. One set of blood cultures was taken at admission and Shigella boydii was isolated. He was treated with intravenous fluids and antibiotics and was discharged on day 7. He was well when reviewed 2 weeks later.

\section{Patient 3}

A 30 year old Melanesian man was admitted to the hospital with a 3 month history of intermittent diarrhoea and weight loss. He also complained of difficulty swallowing and an unproductive cough. At admission, he was wasted, pale and ill. He had oral candidiasis. His weight was $44 \mathrm{~kg}$. He was apyrexial with a pulse rate of $100 /$ minute. There was no palpable lymphadenopathy. His blood pressure was $90 / 70 \mathrm{mmHg}$. Abdominal examination was unremarkable apart from tenderness on deep palpation. Routine haematology and biochemistry findings revealed hypokalaemia. A chest $\mathrm{X}$-ray was normal. No ova, cysts or parasites were seen in repeated stool samples. Blood cultures taken at admission grew Shigella flexneri type 1. A blood slide for malaria and a Widal test were both negative. Human immunodeficiency virus (HIV) serology was negative. Sputum smear and culture were both negative for acid-fast bacilli. A Mantoux test was non-reactive.

The management included intravenous fluids, potassium supplements and intravenous chloramphenicol. There was an initial response but on day 10 his diarrhoea returned accompanied by diffuse abdominal pain. Stool culture did not grow any pathogen and microscopy did not reveal any parasites. The Shigella flexneri proved resistant to chloramphenicol, but sensitive to gentamicin which was commenced. There was, however, little improvement in his condition. On day 24 , endoscopy and sigmoidoscopy were carried out. There was oesophageal candidiasis and multiple submucosal haemorrhagic areas were seen in both rectum and sigmoid colon. No ulcers were seen. Biopsies from the oesophagus confirmed candidiasis but those from the rectum did not show any significant pathology. The condition of the patient continued to deteriorate with profuse diarrhoea. He died following a cardiac arrest and unsuccessful resuscitation. Permission for a postmortem examination was refused.

\section{Discussion}

The clinical implications of bacteraemia in the cases described above are unclear. In the first two cases it appears that the presence of Shigella in the blood was not associated with any clinical manifestations that one would consider unusual in a case of shigellosis. There was no evidence of underlying illness. In the third case the presence of septicaemia may have been related to the general debilitation of the patient from an undiagnosed systemic illness.

Shigella infections are usually restricted to the intestine. The organisms are locally invasive, probably due to the effects of an enterotoxin on epithelial cells. Shigella bacteraemia seems to be a very rare accompaniment of shigellosis. In a review of the literature in 1964, Faucon and Ducloux found 101 cases described between 1912 and $1962 .^{5}$ Since then there have been around 150 further cases described, of which 41 have been in adults. Several studies have demonstrated that when Shigella bacteraemia occurs in adults it is usually associated with underlying disease. ${ }^{6,7}$ Malnutrition was the principal associated feature in Struelens' series from Bangladesh. ${ }^{6}$ Other conditions which have been associated include: diabetes, leukaemia, sickle cell anaemia, malignancy, cirrhosis, immunosuppression and HIV infection. ${ }^{8-10}$ Struelens and his co-authors in their review of 2,018 patients with shigellosis found a higher case fatality rate in patients of all ages with bacteraemic $(21 \%)$ rather than non-bacteraemic shigellosis $(10 \%){ }^{6}$ 
Baskin described five cases of Shigella flexneri bacteraemia occurring in adult patients with HIV infection. ${ }^{9}$ All of these patients had prolonged diarrhoea and colitis despite appropriate antimicrobial therapy. Blaser reported a case of recurrent Shigella flexneri type 2a complicating HIV infection which he attributed to relapse rather than repeated infection. ${ }^{8} \mathrm{He}$ demonstrated the presence of species-specific IgA and IgG in serum taken prior to the onset of diarrhoea. There was no secondary rise in antibody titre during the course of the illness. The failure of serum and faecal antibody to protect against relapse stimulated the authors to question whether cytolytic $\mathrm{T}$ lymphocytes might be involved in the elimination of infected colonic epithelial cells in immunocompetent individuals. Antibody-dependent cell-mediated activity against

\section{References}

1. Haltalin, K.C. \& Nelson, J.D. Coliform septicaemia complicating shigellosis in children. JAMA 1965, 192: 97-99.

2. Gillies, R.R. Shigella; medical microbiology. In: Cruickshank, R.C., Duguid, J.P., Marmoin, B.P. \& Swain, R.H.A. (eds) The Practice of Medical Microbiology, 12th ed., Vol. 11. Churchill Livingstone, London, Edinburgh, 1975, pp. 162164.

3. Isenberg, H.D., Washington, J.A., Barlows, A. \& Sommenwirth, A.C. Collection, handling and processing of specimens. In: Lenette, E.H., Spandling, E.H. \& Truant, J.P. (eds) Manual of Clinical Microbiology. American Society for Microbiology, Washington, DC, 1974, pp. 59-62.

4. Bailey, W.R. \& Scott, E.G. A Textbook for the Isolation of Pathogenic Microorganisms, 5th ed. C.V. Mosby, St Louis, Missouri, 1978.

5. Faucon, R. \& Ducloux, M. Septicemies a Shigella. Med Trop 1964, 24: 537-545.

6. Struelens, M.J., Patte, D., Kabir, I., Salam, A., Nath, S.K. \& Butler, T. Shigella septicaemia: Prevalence, presentation, risk factors and outcome. J Infect Dis 1985, 152: 784-790.
Shigella has been demonstrated in vitro. ${ }^{11}$ The possibility that cell-mediated immunity has a role in both the limitation of intestinal infection and in preventing bacteraemia is an attractive, but unproven, hypothesis.

Blood cultures are not a routine investigation in a patient with dysentery and this may account for the apparent low incidence of bacteraemia in Shigella infection. The serendipitous detection of three cases of Shigella bacteraemia in adults in Port Moresby General Hospital suggests that Shigella bacteraemia may be much more common than the paucity of case reports would suggest. There is increasing evidence to suggest that when Shigella is isolated from the blood, a thorough search for an underlying cause of immunosuppression should be made.

7. Morduchowicz, G., Huminer, D., Siegman-Igra, Y., Drucker, M., Block, C.S. \& Pitlik, S.D. Shigella bacteraemia in adults. Arch Intern Med 1987, 147: 2034-2037.

8. Blaser, M.J., Hale, T.L. \& Formal, S.B. Recurrent shigellosis complicating human immunodeficiency virus infection: failure of pre-existing antibodies to confer protection. $\mathrm{Am} \mathrm{J}$ Med 1989, 86: 105-107.

9. Baskin, D.H., Lax, J.D. \& Barenberg, D. Shigella bacteraemia in patients with the acquired immune deficiency syndrome. Am J Gastroenterol 1987, 82: 338-341.

10. Mandell, W. \& Neu, H.C. Shigella bacteraemia in adults JAMA 1986, 255: 3116-3117.

11. Lovall, G.H., Macdermott, R.P., Summers, P.L., Reeder, A.A., Bertovich, M.J. \& Formal, S.B. Antibody-dependent cell-mediated activity: $\mathrm{K}$ lymphocytes, monocytes and granulocytes are effective against Shigella. J Immunol 1980, 125: 2778-2784. 